\title{
La biblioteca virtual especializada de los derivados de la caña de azúcar: consideraciones, experiencias y perspectivas
}

\author{
Maby Hernández Curbelo*, Rafael Hurtado Vargas*
}

\begin{abstract}
Resumen: En este trabajo se describen los resultados del proyecto de diseño e implementación de la biblioteca virtual especializada sobre los derivados de la caña de azúcar. Se abordan consideraciones, arquitectura de trabajo en ambiente de Internet y características básicas del software para la administración de su colección digital. Esta biblioteca es soporte para la investigación sobre los derivados de la caña de azúcar y constituye el paso inicial hacia la creación de un centro virtual especializado de información y conocimiento para el sector azucarero.
\end{abstract}

Palabras clave: bibliotecas virtuales, administración de bibliotecas, software, ciencias de la información.

\section{A specialized virtual library for sugarcane by-products: considerations, experiences and perspectives}

\begin{abstract}
In this paper, the results of a project for the design and implementation of a specialized virtual library for sugar cane by-products are presented, including initial considerations, an architecture for working in a web environment, and the basic characteristics of software for managing a digital collection. This library supports research into sugar cane by-products and also represents an initial step toward the creation of a specialized virtual centre of information and knowledge for the sugar industry.
\end{abstract}

Keywords: virtual libraries, virtual library management, software, information sciences.

\section{Introducción}

En el marco de las condiciones actuales de desarrollo se hace necesario, para la realización de las investigaciones científicas, la producción industrial y la capacitación, el uso eficiente de la información disponible en las temáticas de interés. Esto implica el conocimiento de la bibliografía disponible en cada área de trabajo y el acceso a esa documentación a texto completo. Para lograrlo, deben desarrollarse tecnologías que lleven los servicios de información al puesto de

" Instituto Cubano de Investigaciones de los Derivados de la Caña de Azúcar (ICIDCA). La Habana, Cuba. Correo-e: maby.hernandez@icidca.edu.cu; rafael.hurtado@icidca.edu.cu.

Recibido: 01-06-20; 2. ${ }^{\mathrm{a}}$ versión: 22-07-2010; aceptado:13-09-2010. 
trabajo, lo cual es posible debido al desarrollo alcanzado por las Tecnologías de la Informática y las Comunicaciones (TIC).

La sociedad moderna se enfrenta a la necesidad de crear nuevas formas de organización de los recursos de información en formato electrónico, capaces de satisfacer a un usuario cada vez más exigente. Con Internet la generación y disponibilidad de una colosal cantidad de información se convirtió en gran reto para los profesionales de la información, que deben encontrar la forma más adecuada de organizar y suministrar al usuario la información que precisa, de manera rápida y sencilla.

Las bibliotecas virtuales representan un esfuerzo para reunir lógicamente grupos de recursos de información afín, presentes en un espacio electrónico ilimitado. Este campo cada vez toma mayor importancia dentro de las áreas de la información, representando una interesante combinación entre aplicaciones, sistemas y teorías tradicionales del manejo de información ordenada y estructurada.

Durante los últimos años, el Instituto Cubano de Investigaciones de los Derivados de la Caña de Azúcar (ICIDCA) ha trabajado en la modernización de su red local y en la aplicación de la tecnología de INTRANET. Este servicio ha permitido colocar al alcance de investigadores y dirigentes del centro la información local necesaria para apoyar las actividades de investigación y dirección.

El trabajo originó el desarrollo de la Biblioteca Virtual de los Derivados de la Caña de Azúcar (BVDCA), que brinda una colección digital de materiales especializados a través de la Intranet del instituto, en su primera etapa. Su finalidad es difundir conocimientos y experiencias para el trabajo de técnicos, investigadores, estudiantes y profesionales del sector, en apoyo a la investigación, producción y dirección. Además, ofrece otros servicios típicos encontrados en la mayoría de las bibliotecas virtuales existentes. Actualmente se trabaja en la integración con otras bibliotecas afines, lo cual contribuirá a la creación de un centro virtual de información y conocimiento, con cobertura nacional e internacional, para el desarrollo de todas las actividades del sector.

\section{Construcción de la biblioteca virtual}

Entre los elementos a considerar para la construcción de una biblioteca virtual destacan la digitalización, los estándares de metadatos, los contenidos y sus tipos, las interfaces de búsqueda y los servicios que se prestan a los usuarios de la misma (Solorio, 2006). Un aspecto de suma importancia en la construcción de una biblioteca virtual será la planificación de la misma, la cual debe considerar estos aspectos:

- Objetivos de la colección digital.

- Características y necesidades de información de los usuarios.

- Tipos de documentos que va a contener.

- Índices para las búsquedas. 
- Clasificadores para la organización de los documentos.

- Formatos de presentación de los resultados de las búsquedas.

- La selección de las mejores técnicas, formatos, software y hardware que se utilizarán en la digitalización de los diversos tipos de información.

- La difusión de la información a través de la red, así como los derechos de autor.

- La gestión de la biblioteca y las funciones de los profesionales encargados de la misma para garantizar que cumpla los objetivos previstos (Faba y Muño, 2004).

Parte esencial de la biblioteca virtual son los objetos digitales, que pueden ser de diversos tipos, formatos, con derechos de autor o de acceso libre. En cada caso es necesario considerar las restricciones de acceso según el tipo de usuario que lo esté intentando y las políticas establecidas a tal efecto a partir de la clasificación de estos objetos digitales.

\section{Arquitectura de la biblioteca virtual}

La figura 1 muestra la arquitectura de referencia para la biblioteca virtual (Sánchez, 2004):

FIGURA 1

Arquitectura de referencia

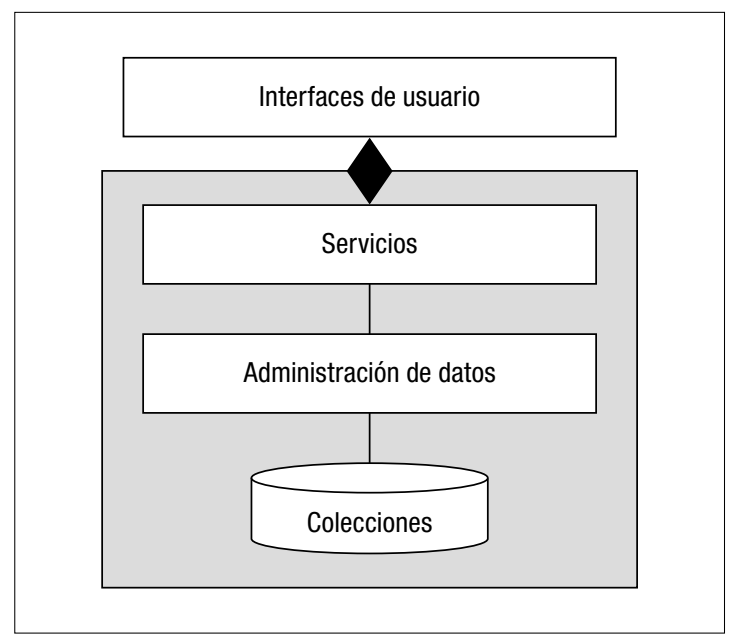

Esta arquitectura general sirve de marco a los desarrollos y consta de cuatro capas o niveles conceptuales. En el nivel conceptual más bajo se encuentra las colecciones, almacenadas en diferentes formatos y dispositivos físicos. Sobre las 
colecciones es necesario contar con un nivel de administración de datos, en el que se incluyen componentes como manejadores de bases de datos relacionales y diversos mecanismos de recuperación de información. La siguiente capa, el nivel de servicios, facilita el manejo de las funciones que ofrece la biblioteca. Finalmente, en el nivel conceptual más alto se ubican las interfaces y ambientes que permiten a los usuarios utilizar y enriquecer las colecciones, así como interactuar con otros usuarios.

Una vista más detallada de la arquitectura para la biblioteca virtual de los derivados puede verse en la figura 2 donde se muestra su diagrama de despliegue.

\section{FIGURA 2}

Arquitectura de la biblioteca virtual de los derivados

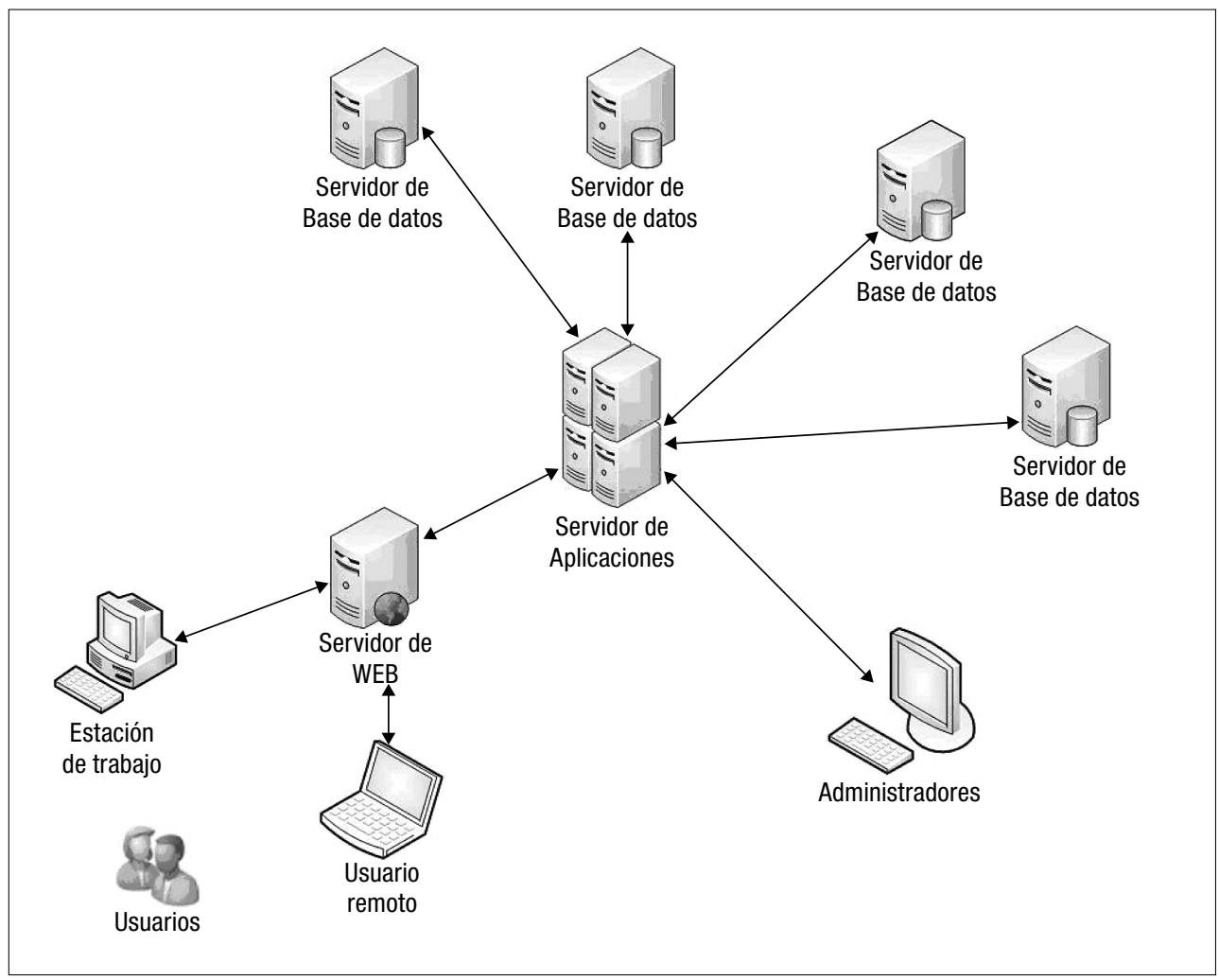

\section{Software para administración de la biblioteca virtual}

Para seleccionar o implementar un software integrado para administrar la biblioteca hay que tener en cuenta cuatro ámbitos: funcional, tecnológico, económico y técnico (Araya y Pérez, 2006) y la participación del bibliotecario es im- 
portante para esta selección de forma conjunta con el personal asociado a las tecnologías de la información (Vasconcelos, 2004). A partir de este análisis es que se determina si un paquete de software cumple los requerimientos o cuales son los módulos que deben implementarse. Existe software propietario comercial que puede adaptarse a necesidades específicas, software libre o de código abierto (Sanjo, 2007; Rodríguez y Peña, 2004; Porcel y Rodríguez, 2005) y hechos a la medida.

El sistema BIBLIO (versión 5.0) es un software propietario implantado en nuestra biblioteca (Hurtado y otros, 1996; Hurtado y otros, 2001). Ha ganado en funcionalidad e incorporado nuevos módulos a lo largo de su ciclo evolutivo, destacando: selección y adquisición, procesamiento de fondos documentales (digitales y no digitales) según la catalogación utilizada en base a las normas cubanas, préstamos, estadísticas y búsquedas. Permite crear diferentes bases de datos en distintas entidades, cuyos contenidos podrán agruparse de acuerdo con sus propiedades y características comunes, las cuales se pondrán en un servidor para su acceso en línea.

Biblio 5.0 cuenta con un módulo de acceso público en línea para usuarios remotos, estadísticas asociadas a estos accesos desde el Web (Babini y otros, 2007) y un módulo de diseminación selectiva de información.

\section{El Web como interfaz de la biblioteca virtual}

La BVDCA es un sitio Web que se crea como puerta de entrada para permitir a los usuarios el acceso remoto a las colecciones y servicios. Su propósito es poner a disposición de técnicos, investigadores, estudiantes y profesionales del sector una colección digital de materiales especializados, para ofrecer su acceso a texto completo a través de la Intranet.

Si bien la tecnología que soporta una biblioteca virtual es de gran importancia, el éxito radica en lo que finalmente se muestra al usuario. Así, se puede abordar este aspecto en dos partes: primero, el diseño global de la página como interfaz para interactuar con el usuario; y segundo, la interfaz de búsque$\mathrm{da}$, que tiene especial importancia por ser el servicio principal de la biblioteca virtual.

Las opciones de búsqueda implementadas son claras (figura 4), el usuario debe saber sobre qué se está buscando y qué libertad tiene para hacer búsquedas complejas, si se hace sobre textos completos o sólo sobre algunos campos; de qué manera pueden formular o articular sus parámetros de búsquedas y qué hacer si no encuentra la información que necesita. Los campos de búsqueda principales son los que tradicionalmente se utilizan en una biblioteca tradicional: palabras claves, título, autor y fecha de publicación.

Los nuevos métodos de organización estructurada de información permiten hacer búsquedas nuevas sobre el texto completo y cualquier otro campo de la ficha bibliográfica. Debe tenerse cuidado de no saturar al usuario con un gran 
FIGURA 3

Página principal de la BVDCA

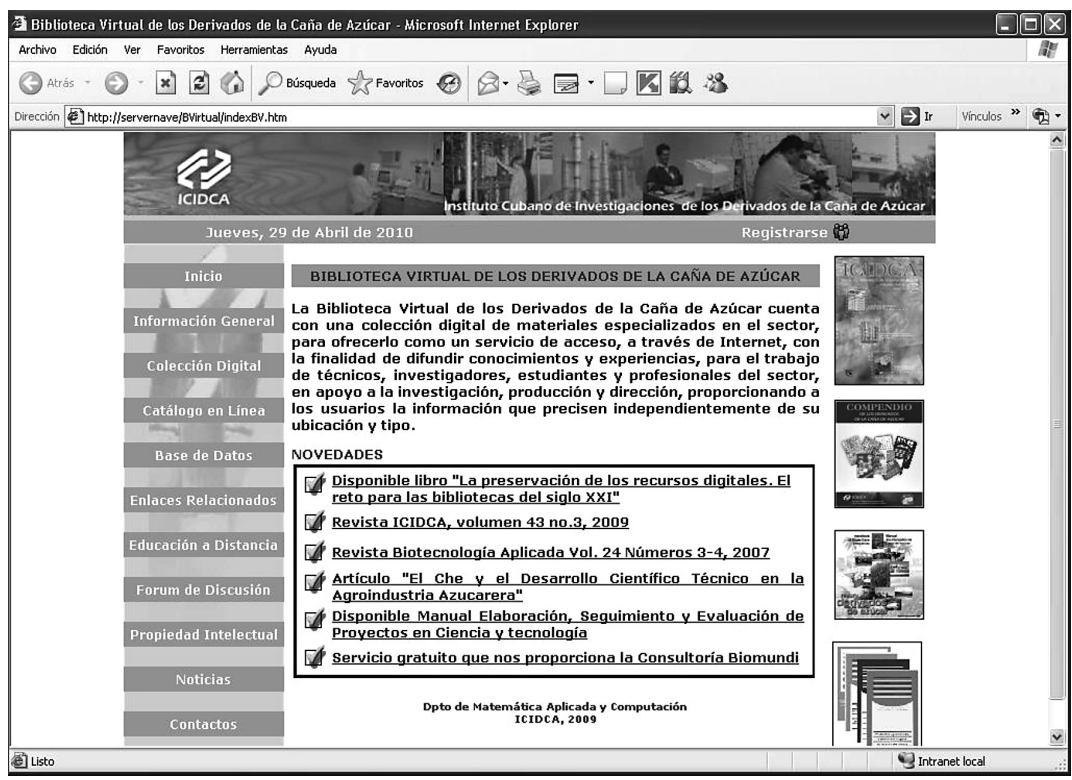

FIGURA 4

Búsquedas en la biblioteca virtual

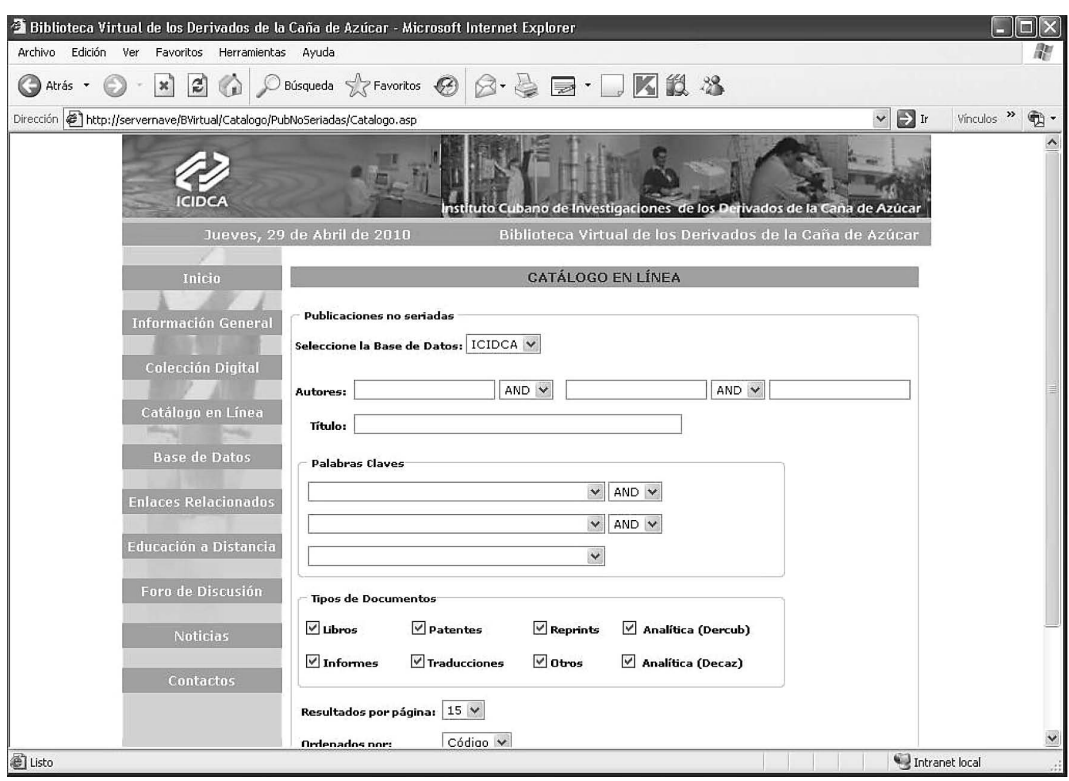


número de opciones de búsqueda, que pueden complicar el proceso y ocasionar que el usuario se sienta abrumado por tantas alternativas.

Para desplegar los resultados de una búsqueda (figura 5) deben considerarse dos factores: la estructura de la información y las necesidades del usuario. Los resultados pueden ser desplegados en orden: cronológico, alfabético por autor, título u otros campos. Para cada documento que forme parte del conjunto resultante de la búsqueda aparece la indicación de si puede ser descargado a texto completo o no, según el control de usuarios establecido.

\section{FIGURA 5}

Resultados de una búsqueda en la BVDCA

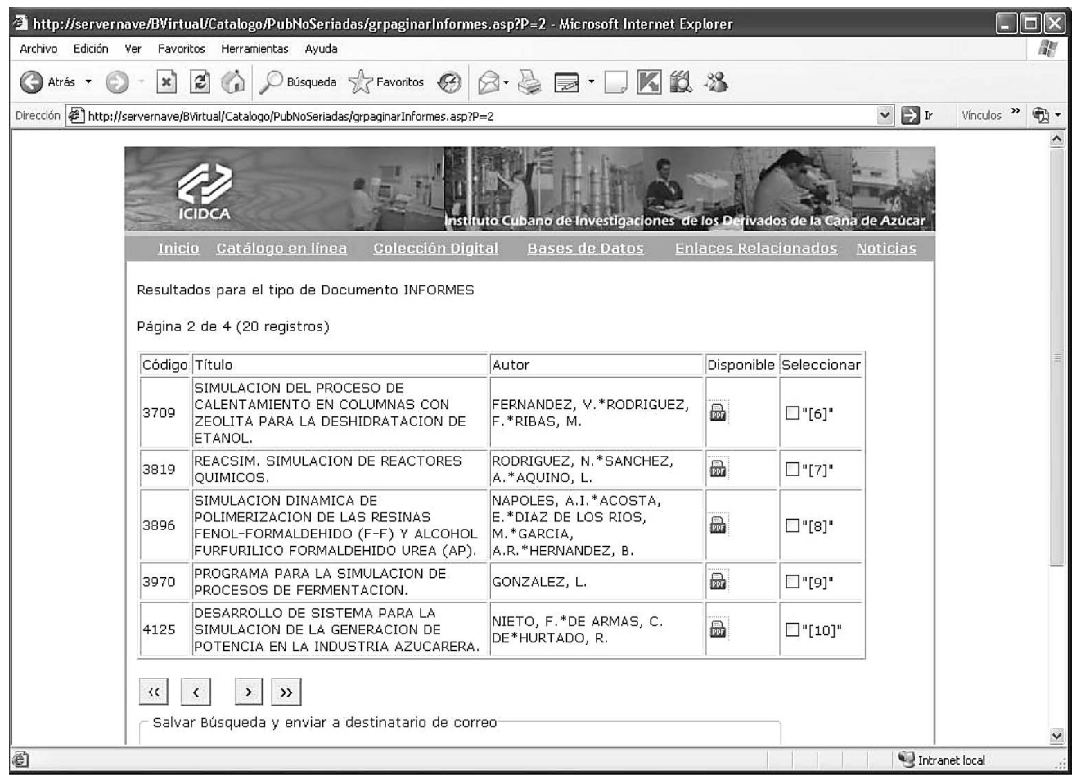

\section{Servicios de la biblioteca virtual}

Existen variados modelos para diseñar e implementar servicios y productos para el Web. Se basan en las propuestas de la arquitectura de la información y la usabilidad, que comprende pasos que van desde el estudio de la comunidad de usuarios hasta la implementación tecnológica y evaluación, sin dejar a un lado la organización de la información y el diseño gráfico y visual de la interface.

La estructura de la biblioteca virtual considera estos modelos en tres dimensiones definidas (Bonilla, 2007):

- Bibliotecológica. Considera los componentes de este tipo de sistema de información: usuarios, servicios y productos de información, elementos de 
organización y representación de contenidos, sistemas de búsqueda y recuperación de información, así como otros recursos.

- Tecnológica. Dimensión que parte de la concepción de una biblioteca digital como un sitio Web, y del análisis del software y hardware disponibles para su implementación.

- Interacción usuario-sistema. Enfocado básicamente hacia el diseño de la interface, traducción visual y funcional del sistema, mediante la cual interactúa el usuario con el contenido.

El servicio por excelencia dentro de la biblioteca es la búsqueda y recuperación de información a texto completo de forma organizada y con documentos estructurados. Según el universo de usuarios que se desea atender se desarrollan servicios adicionales, consulta de catálogos, vínculos a sitios relacionados con el tema de especialización o hacia otras bibliotecas y las listas de discusión como un buen foro para formar grupos de usuarios activos con intereses comunes. Se incluyen además, noticias y eventos referentes a temas de interés.

También se puede proporcionar la tradicional diseminación selectiva de la información por suscripción, en la que los usuarios pueden recibir, mediante correo electrónico, la información de su interés o los enlaces a páginas que los lleven a ella.

La gama de servicios puede ser tan amplia como sea la imaginación del bibliotecario o coordinador del proyecto, pero no hay que olvidar el objetivo principal de ofrecer información estructurada y organizada.

\section{Trabajos futuros}

El trabajo futuro se centrará en las siguientes etapas que forman parte del proyecto:

- Integrar otras bibliotecas del sector en este proyecto de biblioteca virtual especializada.

- Adecuar, sobre la base de esta integración, las políticas de acceso a la información y los tipos de usuarios que existirán.

- Mantener la colección digital actualizada a partir del análisis estadístico del uso de la biblioteca digital, una vez que tenga cobertura nacional e internacional.

\section{Conclusiones}

- El diseño e implementación de la biblioteca virtual especializada de los derivados de la caña de azúcar, con una amplia colección digital en constante actualización, es un elemento importante para el desarrollo de la actividad científica en este campo. 
- La biblioteca virtual especializada de los derivados puede ser accesada en línea, lo cual presupone la existencia de mecanismos para control de autoridades, las cuales tendrán los niveles de acceso según la política establecida para los documentos de la colección digital.

- El software de administración garantiza el análisis estadístico para lograr una acertada toma de decisiones. Esto es determinante para la actualización de la colección digital y la satisfacción de las necesidades de información de los usuarios.

- La implementación de un módulo de diseminación selectiva de información contribuye a mantener actualizados a los usuarios de la biblioteca virtual con las informaciones relevantes y recientes de acuerdo a sus necesidades.

\section{Bibliografía}

Araya, A., y Pérez, C. (2006). Aplicación de un algoritmo logístico para la selección de un software integrado de bibliotecas. Serie Bibliotecología y Gestión de Información, (14), 1-64. Disponible en: http://eprints.rclis.org/6433/1/serie14.pdf [enero de 2010].

Babini, D.; Vergara, F., y Archuby, G. (2007). Estadísticas de usuarios en una biblioteca virtual. El caso de la biblioteca virtual de la red Clacso. El profesional de la información, vol. 16 (1), 57-61. Disponible en: http://www.elprofesionaldelainformacion.com/ contenidos/2007/enero/07.pdf [enero de 2010].

Bonilla, K. (2005). De la biblioteca virtual hacia centros de información y conocimiento virtual: Estudio de caso de la universidad técnica de Delft. 7mo Congreso ISKO-Espa$\tilde{n} a$, 91-104. Barcelona. Disponible en: http://bd.ub.es/isko2005/bonilla.pdf [enero de 2010].

Faba, C., y Muño M. V. (2004). La nueva gestión en las bibliotecas virtuales. Boletín de la Asociación Andaluza de Bibliotecarios, (74), 19-28. Disponible en: http://www.aab. es/pdfs/baab74/74a2.pdf [enero de 2010].

Hurtado, R.; Carnesoltas, A., y Roselló, S. (1996). BIBLIO: Un sistema para gerencia de bibliotecas. Revista Española de Documentación Científica, vol. 19 (3), 327-335.

Hurtado, R.; Hernández, M., y Roselló, S. (2001). Sistema para gerencia de bibliotecas (BIBLIO Versión 3.0). Revista ICIDCA, vol. 35 (2-3), 42-45.

Porcel, M. L., y Rodríguez, M. (2005). Software libre: una alternativa para las bibliotecas. Acimed, vol. 13 (6). Disponible en: http://bvs.sld.cu/revistas/aci/vol13_6_05/aci090605. htm [febrero de 2010].

Rodríguez, M., y Peña, R. (2004). CLABEL: un software libre para la creación de catálogos públicos con acceso en línea en las bibliotecas. Acimed, vol. 12 (2). Disponible en: http://bvs.sld.cu/revistas/aci/vol12_2_04/aci02204.htm.

Sánchez, J. A. (2004). U-DL-A: Bibliotecas digitales en la UDLA. Revista Digital Universitaria, vol. 5 (6), 2-15. Disponible en: http://www.revista.unam.mx/vol.5/num6/art33/ art33.html [enero de 2010].

Sanjo, J. (2007). Adoption of open source digital library packages: a survey. Proceedings CALIBER 2007: 5th International Convention on Automation of Libraries in Education and Research Institutions, 98-102. Chandigarh, India. Disponible en: http://www. 
eprints.org/community/blog/index.php?/archives/191-Adoption-of-Open-Source-Digital-Library-Software-Packages-a-Survey.html [enero de 2010].

Solorio, J. (2006). La digitalización, metadatos y contenidos en las bibliotecas virtuales. Memorias Congreso Internacional de Información INFO'2006, La Habana, Cuba. Disponible en: http://www.congreso-info.cu/resumeninfo06 [febrero de 2010]

Vasconcelos, G. (2004). Participacao do bibliotecario na criacao e planejamento de projetos de softwares: o envolvimento com a tecnologia da informação. BIBLIOS, vol. 5 (17). Disponible en: http://dici.ibict.br/archive/00000246/01/RDBCI-2004-24.pdf [febrero de 2010]. 